\title{
Paleoclima de La Guajira, Colombia; según los anillos de crecimiento de Capparis odoratissima (Capparidaceae)
}

\author{
Jorge Andrés Ramírez ${ }^{1} \&$ Jorge Ignacio del Valle ${ }^{2}$ \\ 1. Département des Sciences Biologiques, Université du Québec à Montréal. Centre d'étude de la Forêt; \\ ramirez_correa.jorge_andres@courrier.uqam.ca \\ 2. Departamento de Ciencias Forestales, Universidad Nacional de Colombia Sede Medellín; jidvalle@unal.edu.co
}

Recibido 20-IX-2010. C Corregido 10-I-2011. Aceptado 14-II-2011.

\begin{abstract}
Paleoclimate of La Guajira, Colombia; by the growth rings of Capparis odoratissima (Capparidaceae). There is great concern about the effect of climate change in arid and subarid areas of the tropics. Climate change combined with other anthropogenic activities such as deforestation, fires and overgrazing can accelerate their degradation and, consequently, the increases in losses of biological and economic productivity. Climate models, both local and global, predict that rainfall in the arid Peninsula of La Guajira in the Colombian Caribbean would be reduced and temperature would be increased as a result of climate change. However, as there are only suitable climate records since 1972, it is not possible to verify if, indeed, this is happening. To try to verify the hypothesis of reducing rainfall and rising temperatures we developed a growth ring chronology of Capparis odoratissima in the Middle Peninsula of La Guajira with 17 trees and 45 series which attain 48 years back. We use standard dendrochronological methods that showed statistically significant linear relationship with local climatic variables such as air temperature, sea surface temperature (SST), annual precipitation and wind speed; we also reach to successful relationship of the chronology with global climatic variables as the indices SOI and MEI of the ENSO phenomenon. The transfer functions estimated with the time series (1955 and 2003) do not showed statistically significant trends, indicating that during this period of time the annual precipitation or temperatures have not changed. The annual nature of $C$. odoratissima growth rings, the possibility of cross-dated among the samples of this species, and the high correlation with local and global climatic variables indicate a high potential of this species for dendrochronological studies in this part of the American continent. Rev. Biol. Trop. 59 (3): 1389-1405. Epub 2011 September 01.
\end{abstract}

Key words: dendroclimatology, annual tree rings in tropical trees, tropical semiarid forest, radiocarbon dating, climate reconstructions.

Contra el mito, jamás demostrado, de inexistencia de anillos anuales que almacenen información ambiental en todos o casi todos los árboles tropicales (Whitmore 1975, Lieberman et al. 1985, Schweingruber 1988, Lamprecht 1990, Baker 2003) se oponen más de cien investigaciones dendrocronológicas llevadas a cabo desde hace más de cien años en los más diversos ambientes tropicales (Roig 2000, Worbes 2002, Brienen 2005, Rozendaal 2010).

Existe gran preocupación por los efectos del cambio global en las zonas áridas del hemisferio norte tropical y subtropical por cuanto se afirma que los procesos de desertificación y degradación se han venido acelerando (Graingeret al.2000) y están representados por pérdida o reducción de productividad biológica o económica así como de complejidad ecosistémica (Brauch \& Spring 2009). Según New et al. (2001) en estas zonas desde la década del 50 la precipitación se ha reducido $6.3 \mathrm{~mm} /$ década. Se afirma que la duración y fortaleza del fenómeno ENSO (El Niño Southern Oscillation) ha venido aumentando (Lyon 2004) lo que 
conlleva alteraciones espaciales y temporales de los patrones de temperatura, precipitación, insolación y vientos (Le Houérou 1996).

Aproximadamente 1\% del territorio colombiano corresponde a zonas áridas o semiáridas, la mayoría de ellas en la península de La Guajira (Le Houérou 1996). Sin embargo, cuando se tiene en cuenta que estas condiciones se extienden hacia Venezuela cubriendo amplios territorios aledaños al golfo de Venezuela y el lago de Maracaibo, se comprende la pertinencia de esta investigación. Los modelos climáticos estiman que en esta región el cambio climático se manifestará acentuándose los eventos climáticos extremos con sequías más rigurosas y prolongadas y, a largo plazo, una marcada aridización (IDEAM 2001, 2008). No obstante, además de que estos modelos se alimentan con registros instrumentales de corta duración y escaso cubrimiento, diversas investigaciones revelan que los modelos de simulación disponibles no aciertan cuando trata de predecir los efectos del cambio climático en las regiones tropicales (Allan \& Soden 2008). En esta investigación nos proponemos identificar la señal climática contenida en los anillos de crecimiento de $C$. odoratissima mediante técnicas dendroclimáticas. Partimos de estas hipótesis: a) los anillos de $C$. odoratissima son anuales, b) el ancho de los anillos varía periódica y similarmente en el conjunto de árboles de la región, c) los anillos son sensibles a las variables climáticas de la región y, por tanto, su anchura permitirá reconstruir el clima antes de la existencia de registros climáticos instrumentales.

\section{MATERIALES Y MÉTODOS}

Área de estudio y datos climáticos: Realizamos el estudio en la parte media de la península de La Guajira, municipios de Riohacha, Manaure y Uribia, entre las coordenadas geográficas aproximadas $11^{\circ} 30-12^{\circ} 05^{\prime} \mathrm{N}$ - $72^{\circ} 20^{\prime}-72^{\circ} 30^{\prime} \mathrm{W}$. La topografía de la zona es plana a suavemente ondulada, formada por arenales y dunas cubiertas por árboles dispersos, achaparrados y con frecuencia espinosos. El sobrepastoreo por ganado caprino y ovino y la erosión eólica han degradado la vegetación y los suelos.

Empleamos los datos climáticos de precipitación pluvial y temperatura del aire entre 1972 y 2003 de la estación hidrometeorológica del IDEAM (Instituto de Hidrología Meteorología y Estudios Ambientales de Colombia) en Manaure $\left(11^{\circ} 46^{\prime} \mathrm{N}-72^{\circ} 27^{\prime} \mathrm{W}\right)$.

La distribución de las lluvias mensuales es bimodal, la precipitación media anual es $397 \mathrm{~mm}$ y la temperatura media anual del aire $28.8^{\circ} \mathrm{C}$. Asimismo, obtuvimos la serie de temperatura superficial del mar (TSM) del ICOADS (The International Comprehensive Ocean-Atmosphere Data Set Projet; Reynolds \& Smith1994) con resolución mensual entre los años 1981-2003 del cuadrante de $1^{\circ}$ por $1^{\circ}$ de las coordenadas $11^{\circ} 30^{\prime}-12^{\circ} 30^{\prime} \mathrm{N}-71^{\circ} 30^{\prime}$ $72^{\circ} 30^{\prime} \mathrm{W}$. Descargamos la serie de velocidad del viento del Reanálisis NCEP-NCAR (Kalnay et al. 1996), 1948-2003, del cuadrante de $1^{\circ} 54^{\prime}$ por $1^{\circ} 54^{\prime}$ localizado entre las coordenadas $10^{\circ} 29^{\prime}-12^{\circ} 23^{\prime} \mathrm{N}-71^{\circ} 15^{\prime}-73^{\circ} 07^{\prime} \mathrm{W}$. Las series mensuales del índice SOI (Southern Oscillation Index; Trenberth 1984) y MEI (Multivariate ENSO Index; Wolter \& Timlin 1998) que cuantifican la actividad del ENSO las obtuvimos en las siguientes páginas web: http://www.cgd.ucar.edu/cas/catalog/climind/ soi.html (NCAR 2007) y http://www.esrl.noaa. gov/psd/people/klaus.wolter/MEI/ (NOAA 2007), respectivamente (Fig. 1).

Especie seleccionada: $C$. odoratissima fue seleccionada por su abundancia en la región, y por la presencia de anillos visibles a simple vista en las secciones transversales pulidas. Esta es una especie siempreverde que alcanza $8 \mathrm{~m}$ de altura y $30 \mathrm{~cm}$ de diámetro.

\section{Recolección, tratamiento de las mues-} tras y anualidad de los anillos: En 2004 y 2005 cortamos secciones transversales de 19 árboles a $1.3 \mathrm{~m}$ del suelo a lo largo del área de estudio, las cuales se secaron al aire. Luego las pulimos con lija desde grado 80 hasta 600 de manera progresiva. Predatamos, bajo el supuesto de anualidad, dos anillos de cada una 


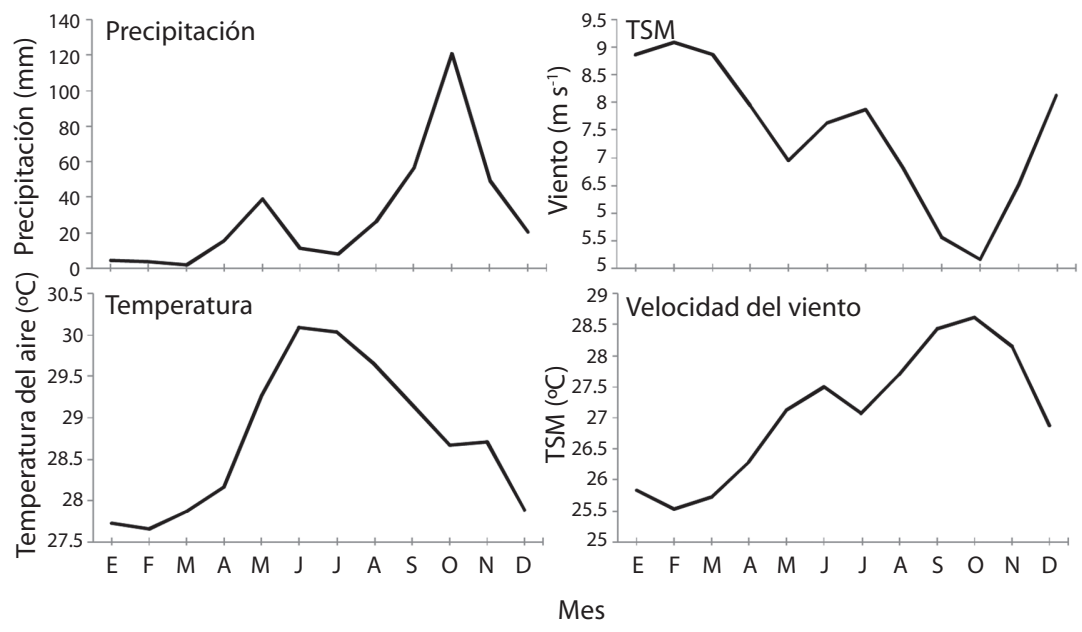

Fig. 1. Ciclo medio anual de algunas series climáticas del área de estudio.

Fig. 1. Mean annual cycle of some climate series in the study area.

de cuatro secciones transversales. Con sierra manual cortamos a cada sección transversal una rodaja de aproximadamente un centímetro de espesor para evitar la posible contaminación con aceite de la motosierra empleada en la corta de la sección transversal. Con una broca extrajimos unos 10-20g de madera de los anillos predatados y enviamos las muestras para el análisis de $\mathrm{C}^{14}$ a cada uno de estos dos laboratorios: Laboratorio de Radiocarbono de Kiev (Código Ki), Ucrania, y Laboratorio de Radiocarbono de la Academia de Ciencias Médicas de Ucrania (Código IHME). Para confirmar la predatación empleamos el efecto de las bombas nucleares en la concentración de radiocarbono en la atmósfera y en la madera de los anillos (Worbes \& Junk 1999, Worbes et al. 2003) y calibramos con el programa CALIBOMB (Hua \& Barbetti 2004, Reimer et al. 2004), en la zona dos correspondiente al trópico norte.

Medición de anillos de crecimiento y elaboración de cronologías: Sobre cada sección pulida dibujamos dos a cuatro radios (series) para un total de 51 series. Sobre cada serie marcamos con lápiz de punta fina los anillos observándolos con estereozoom gradual hasta
$35 \mathrm{X}$ y posteriormente, para evitar errores en la marcación, conectamos la circunferencia de los anillos cada cinco años. En una plataforma Velmex medimos el ancho de los anillos sobre los radios marcados y perpendicularmente a su límite con precisión de $0.001 \mathrm{~mm}$. Llevamos a cabo la datación cruzada y evaluamos los posibles errores mediante el programa COFECHA (Holmes 1983, Grissino-Mayer 2001). Este programa sincroniza cada serie comparándola con una cronología maestra de referencia ajustada mediante funciones spline cúbicas que filtra las señales de baja frecuencia debidas al crecimiento de los árboles.

Estandarizamos y construimos las cronologías de índice de ancho de los anillos mediante el programa ARSTAN (Cook \& Holmes 1996). ARSTAN elimina la tendencia ontogénica debida a la disminución del ancho de los anillos con la edad usando funciones exponenciales negativas o lineales. Luego, mediante funciones spline cúbicas, removimos $50 \%$ de la amplitud de la señal en una longitud equivalente al $67 \%$ de la longitud media de la serie. Este procedimiento tiende a filtrar el ruido producido por señales de baja frecuencia en el ancho de los anillos preservando la señal 
climática de alta frecuencia anual o subanual (Cook \& Kairiukstis 1992). Para disminuir el efecto de valores extremos empleamos la media robusta doblemente ponderada de los residuos. Luego aplicamos un modelo autoregresivo para obtener una cronología residual sin autocorrelación temporal en las series que usamos en los modelos estadísticos de respuesta climática. ARSTAN, AutoRegressiveSTANdarization de Cook \& Holmes (1996) genera diversos estadísticos tales como la relación señal ruido $(S N R)$ que expresa la fortaleza de la señal común entre los árboles y la sensibilidad media $(M S)$, que expresa las diferencias relativas entre anillos contiguos.

Análisis de la respuesta climática: Evaluamos el grado de asociación entre las variables climáticas y el crecimiento con el coeficiente de correlación de Pearson y regresión múltiple sobre los componentes principales de los predictores climáticos o función respuesta (Fritts 1976, Blasing et al. 1984). Así, el índice de los anillos de crecimiento constituye la variable dependiente y los datos climáticos mensuales la independiente. Para disminuir la intercorrelación o colinearidad transformamos las variables predictoras mensuales en un nuevo conjunto de variables ortogonales incorrelacionadas (autovectores-eigenvalues- o componentes principales). Dado que el crecimiento durante un año puede estar influenciado por el clima del año anterior, nuestro análisis comprendió desde septiembre de la estación previa de crecimiento hasta agosto del año corriente en que se formaron los anillos. Nuestro año fenológico se inicia en septiembre debido a que, como se muestra en la Fig. 1, el período lluvioso se inicia en este mes. Consideramos los cuatro trimestres: diciembre-enero-febrero (DEF), marzo-abril-mayo (MAM), junio-julioagosto (JJA), y septiembre-octubre-noviembre (SON). Luego ajustamos regresiones simples entre la cronología del ancho de los anillos en función de las variables climáticas para evaluar su tendencia en el largo plazo (funciones de transferencia).

Con el fin de detectar señales cíclicas (componentes armónicos) que aparecen reiteradamente en la cronología residual de anillos, llevamos a cabo un análisis espectral de la serie de tiempo con la trasformada de Fourier.

\section{RESULTADOS}

Caracterización y periodicidad de los anillos de crecimiento: Los anillos se diferencian por porosidad semicircular y bandas de parénquima terminal (Fig. 2).

Los resultados de la datación de los anillos presentados en la Fig. 3 muestran que la probabilidad de acertar con la edad de los ocho



Fig. 2. Sección transversal de Capparis odoratissima y anillos de crecimiento cuyo límite lo marcan bandas de parénquima paratraqueal. Las flechas indican el límite de los anillos.

Fig. 2. Cross section of Capparis odoratissima and growth rings whose limits are marked by paratracheal parenchyma bands. Arrows indicate the limit of the rings. 


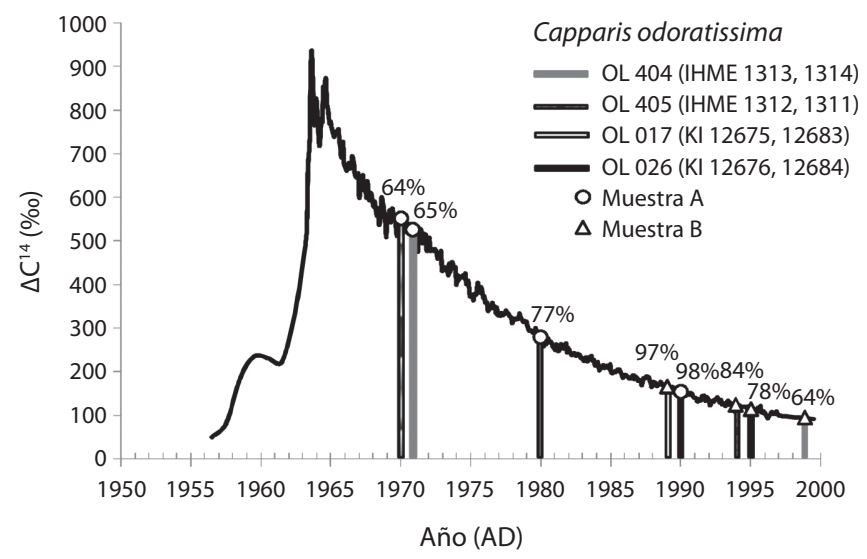

Fig. 3. Análisis de $\mathrm{C}^{14}$ en las muestras de anillos de Capparis odoratissima con el uso CALIBOMB para la calibración. Fig. 3. ${ }^{14} \mathrm{C}$ analysis of tree-ring wood samples from Capparis odoratissima dated using CALIBOMB for calibration.

anillos predatados con los análisis de $\mathrm{C}^{14}$, varió entre 64 y 98\%. También resultó coherente la secuencia entre los dos anillos de cada muestra. Estos resultados son una evidencia muy contundente de la anualidad de estos anillos de crecimiento. En la discusión presentamos evidencias adicionales de la anualidad de los anillos de crecimiento de esta especie.

\section{Cofechado de las series y caracteriza-} ción de las cronologías: En general, los meses de SON de la región estudiada corresponden a un período ecológicamente húmedo (Fig. 1) con precipitaciones mensuales $\geq 100 \mathrm{~mm}$. Ello, aparentemente, induce la actividad cambial formándose un anillo de crecimiento. Los demás meses del año son, en promedio, ecológicamente secos $(<60 \mathrm{~mm})$, en los cuales, probablemente los árboles crecen poco o nada. Este patrón debe desencadenar periodicidad anual del crecimiento, lo que se refleja en la similitud entre las series de crecimiento de los diferentes árboles muestreados. La correlación promedia entre los índices del ancho de los anillos de $C$. odoratissima fue 0.619 con valores individuales entre 0.427 y 0.827 todos estadísticamente significativos $(\mathrm{p}<0.05)$; el porcentaje de sincronización fue $60 \%$. De ello dedujimos la existencia de una señal ambiental común entre todos los árboles. Adicionalmente, con el fin de documentar estadísticamente la correspondencia del crecimiento entre árboles, y señalar el potencial de la especie para estudios dendrocronológicos, se presenta el Cuadro 1 con las estadísticas descriptivas de la serie y la cronología residual.

En la Fig. 4 presentamos la cronología residual. Según las estadísticas descriptivas de

\section{CUADRO 1}

Principales características dendrocronológicas de Capparis odoratissima

\section{TABLE 1}

Capparis odoratissima main dendrochronological characteristics

\begin{tabular}{lc}
\multicolumn{1}{c}{ Período } & 1955 - 2003 \\
Número de árboles & 19 \\
Número de series & 51 \\
Número de anillos & 863 \\
Ancho medio de los anillos (mm) & 3.032 \\
Desviación estándar del ancho de & 1.953 \\
anillos (mm) & \\
Sensibilidad media & 0.451 \\
Relación señal-ruido & 9.963 \\
Autocorrelación de primer orden & -0.111 \\
Varianza del primer vector (\%) & 49.58
\end{tabular}




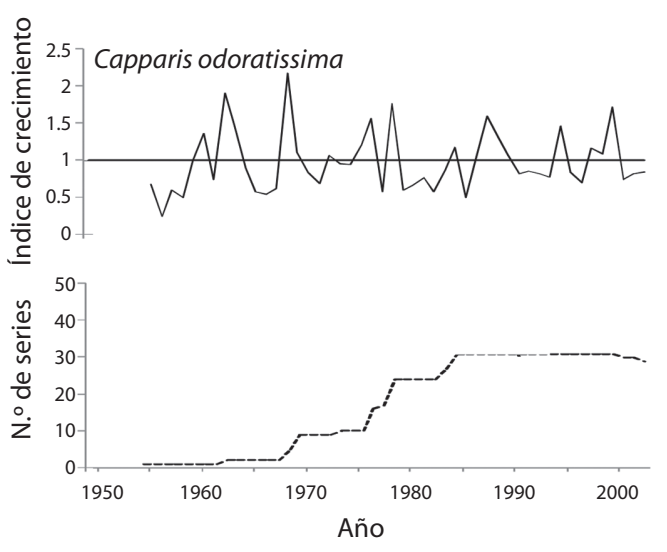

Fig. 4. Arriba, cronología residual del índice de ancho de anillos de crecimiento de Capparis odoratissima. Abajo el número de series empleadas en la cronología.

Fig. 4. Up, residual chronology of ring width indexes of Capparis odoratissima. Down, the number of samples used in the chronology.

la serie, una sensibilidad media superior a 0.4, es relativamente alta (Fritts 1976) lo que implica alta variabilidad en el crecimiento causada por el clima. La baja y estadísticamente no significativa autocorrelación de primer orden (-0.111) significa que los valores sucesivos de la serie tienen baja correlación; por tanto, existe alta varianza de alta frecuencia presumiblemente debida a la variabilidad climática anual o subanual. La relación señal ruido (SNR por su nombre en inglés), aunque no es muy alta (9.993), es comparable con la encontrada en otras especies con tamaños muestrales similares (Cook \& Kariukstis 1992). El análisis de componentes principales señala que gran parte de la varianza del crecimiento de los árboles (superior a 49\%) se expresa en el primer autovector, el cual representa el patrón de anomalías más importante en el comportamiento de los datos originales: entre mayor sea el porcentaje de varianza acumulada en el primer autovector, mayor será la probabilidad de que los primeros componentes principales representen variaciones macroclimáticas de la región.
Correlación entre el ancho de los anillos y los predictores climáticos regionales: $\mathrm{La}$ proporción de la variabilidad de la cronología residual explicada por los modelos de variables climáticas locales calculadas mediante funciones respuesta, fue en la mayoría de los casos, alta y significativa, con $\mathrm{r}^{2}$ de $59.415 \pm 0.222$ para la precipitación, $31.885 \pm 0.267$ para la temperatura del aire, $63.248 \pm 0,146$ para la TSM y de $45.265 \pm 0.277$ para la velocidad del viento, respectivamente. Llama la atención que la TSM explica mejor el crecimiento de C. odoratissima que cualquier otra variable climática local.

Como cabría esperar en ecosistemas áridos, existe correlación positiva y significativa entre el crecimiento y la precipitación pluvial concentrada entre agosto y noviembre (Fig. 5). Este período coincide con la estación lluviosa SON cuyo aporte supera $65 \%$ de la precipitación promedia anual (Fig. 1). Por cuanto la especie también es sensible a la precipitación de agosto de la estación previa, interpretamos que, al menos en algunos años, las lluvias empiezan a caer desde dicho mes activando el cámbium de $C$. odoratissima .

El aumento de la temperatura del aire genera un efecto adverso en el crecimiento mostrado por la correlación negativa durante todo el período considerado (Fig. 5). La correlación es significativamente negativa durante los meses octubre, marzo y abril de ambas estaciones de crecimiento; durante junio de la estación previa y noviembre de la estación de crecimiento. Estos resultados sugieren la existencia de influencia estacional del período MAM, de la estación de crecimiento anterior y de los períodos SON y MAM de la estación de crecimiento actual. En el período MAM la temperatura aumenta rápidamente y durante SON disminuye abruptamente (Fig. 1) reduciendo el crecimiento de los árboles.

Contrario a la temperatura del aire, existen correlaciones positivas y negativas entre el crecimiento y la TSM (Fig. 5), que son significativamente negativas entre marzo y mayo de la estación de crecimiento actual y positiva 

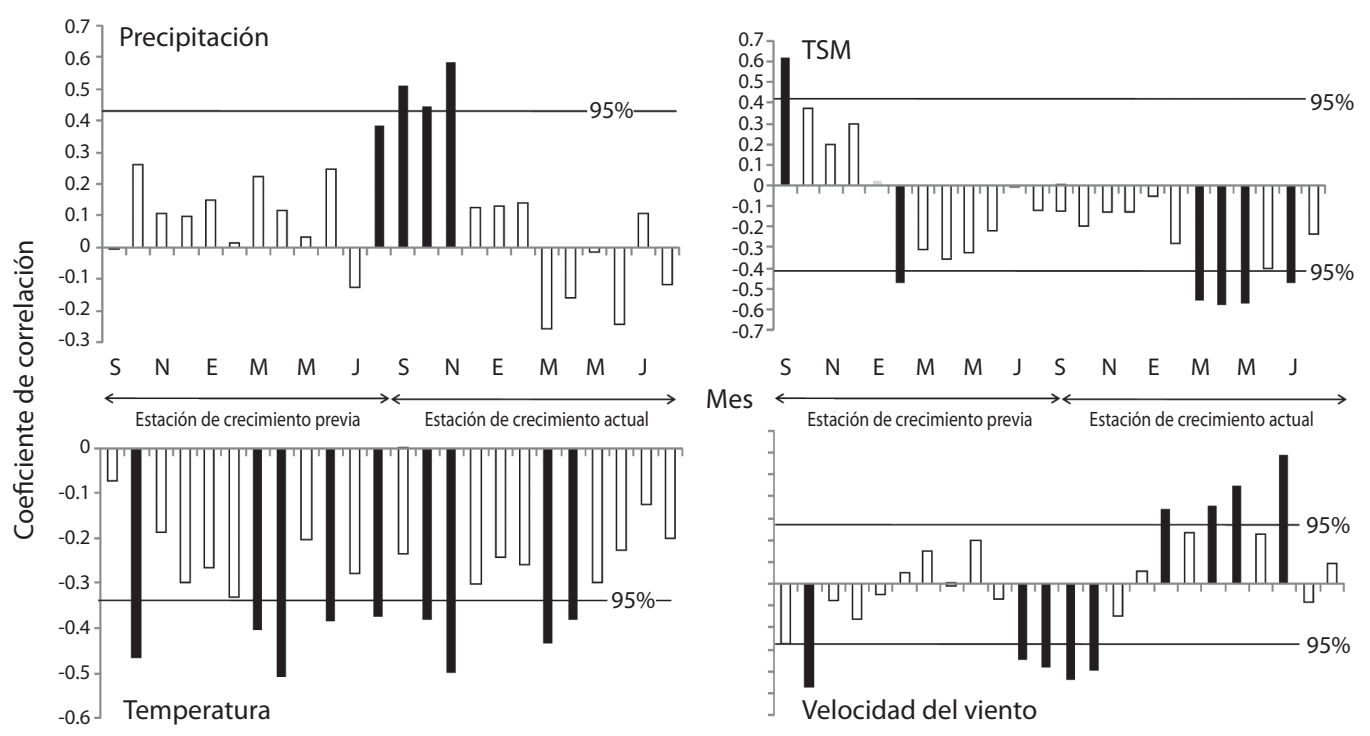

Fig. 5. Coeficientes de correlación mensual entre la cronología residual de Capparis odoratissima y las variables climáticas locales para la estación de crecimiento actual y previa. Las barras negras indican significancia para $\mathrm{p}<0.05$.

Fig. 5. Monthly correlation coefficients between the residual chronology of Capparis odoratissima and local climate variables for the current growing season and a year lagged. The blackbars mean significant for $\mathrm{p}<0.05$.

y negativa, respectivamente, en septiembre y febrero de la estación de crecimiento previa.

La velocidad del viento, cuyo comportamiento es opuesto a la TSM (Fig. 1), presenta correlaciones negativas al inicio de la estación previa siendo significativa en octubre (Fig. 5). Correlaciones significativamente negativas entre julio y octubre cubren ambas estaciones, y positivas, usualmente significativas, desde enero hasta junio de la estación de crecimiento. Por tanto, inicialmente el crecimiento aumenta a medida que se reduce la velocidad del viento, posteriormente este proceso se invierte y tanto el crecimiento como la velocidad del viento aumentan simultáneamente entre enero y junio de la estación de crecimiento.

Funciones de transferencia: En el Cuadro 2 presentamos las regresiones empleadas para estimar las variables climáticas $(Y i)$ en función de la cronología de los anillos de crecimiento $(X i)$. Se nota que la cronología de $C$. odoratissima explica con notable confiabilidad las variables correspondientes a la precipitación total y durante SON así como la TSM. La temperatura del aire y la velocidad del viento no arrojaron ajustes satisfactorios. Con las regresiones significativas de precipitación y TSM estimamos los valores anuales correspondientes a estas variables climáticas entre los años 1955 y 1972, cuando se inició la serie de anillos de crecimiento. Luego ajustamos regresiones lineales entre las variables climáticas en función del tiempo con el fin de evaluar su tendencia. Contrario a lo afirmado por la literatura y a lo esperado para una región árida del trópico norte, ninguna de las variables climáticas mostró para el período 1955-2003 una diferencia significativa con la media que evidenciara tendencias referentes a cambio en los patrones de precipitación y TSM en la media península de La Guajira. En la Fig. 6 se observa la tendencia correspondiente a la precipitación total y durante SON. No se muestra la correspondiente a la TSM. 


\section{CUADRO 2}

Funciones de transferencia de las variables climáticas regionales

TABLE 2

Transfer function of the regional climate variables

\begin{tabular}{llcc}
\multicolumn{1}{r}{ Variable $\left(Y_{i}\right)$} & \multicolumn{1}{c}{ Regresión } & $r^{2}$ ajustado $(\%)$ & Probabilidad \\
Precipitación total $(\mathrm{mm})$ & $Y_{t}=91.2301+265.383 X_{t}$ & 56.553 & 0.0000 \\
Precipitación SON $(\mathrm{mm})$ & $Y_{t}=-19.326+231.379 X_{t}$ & 59.483 & 0.0000 \\
Temperatura del aire $\left({ }^{\circ} \mathrm{C}\right)$ & $Y_{t}=29.205-0.473 X_{t}$ & 6.840 & 0.0840 \\
TSM $\left({ }^{\circ} \mathrm{C}\right)$ & $Y_{t}=27.441-0.378 X_{t}$ & 24.524 & 0.0153 \\
Viento $(\mathrm{m} / \mathrm{s})$ & $Y_{t}=7.576-0.1443 X_{t}$ & 0.580 & 0.2685
\end{tabular}

En negrita las regresiones significativas. / In bold significant regressions.

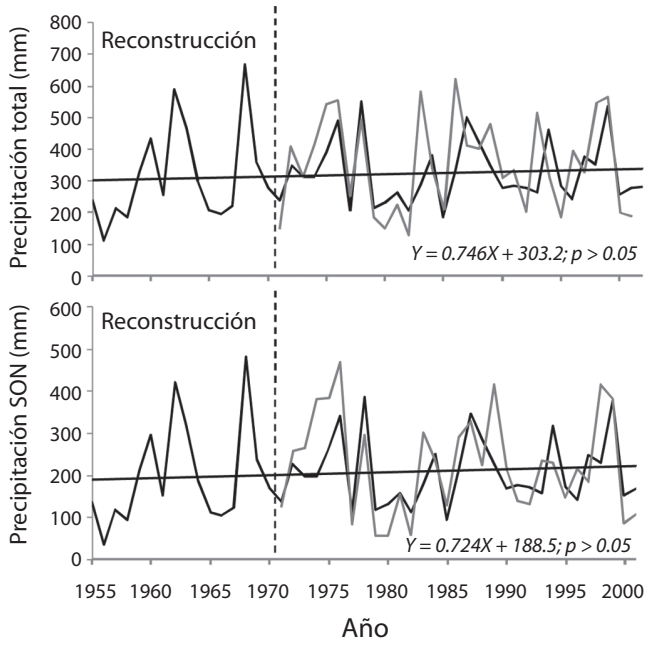

Fig. 6. Reconstrucción de la precipitación total y durante la estación SON $\left(Y_{t}\right)$ por medio del ancho de anillos $\left(X_{t}\right)$ de Capparis odoratissima. Valores observados en gris y reconstruidos en negro. Las ecuaciones presentan las líneas de tendencia de las precipitaciones en función del tiempo. Fig. 6. Reconstruction of the total annual rainfall and rainfall during September to November $\operatorname{SON}\left(Y_{t}\right)$ depending on the width of rings $\left(X_{t}\right)$ of Capparis odoratissima. Gray: observed values; black: reconstructed values. The equations show the trend lines of the rainfalls as a function of time.

Con el fin de evaluar el modelo desarrollado para la precipitación anual y la precipitación durante SON (Fig. 6), se utilizó un período aislado de precipitación de la estación hidrometeorológica del IDEAM en la ciudad de Manaure (11 $\left.{ }^{\circ} 46^{\prime} \mathrm{N}-72^{\circ} 47^{\prime} \mathrm{W}\right)$ entre 1956 y 1965 para verificación. Para verificar el modelo encontrado se utilizó el coeficiente de correlación de Pearson y la prueba $t$-student (Fritts 1976, Cook \& Kairiukstis 1992).

Las correlaciones entre los datos reales y reconstruidos de la precipitación total y SON son de 0.84 y 0.72 , respectivamente (ambas correlaciones significativas al $0.05 \%$ ). La prueba de $t$ resultó significativa para $=0.05$ en ambos casos indicando que no existe diferencia significativa entre ambas muestras.

\section{Correlación entre el ancho de los anillos} y los predictores climáticos globales: En cuanto a los componentes espectrales, la cronología no se diferenció significativamente de ruido blanco $(\mathrm{p}<0.05)$; esto es, ruido de origen aleatorio, situación previsible en series a las que se les ha eliminado la autocorrelación temporal. No obstante, se detectaron dos señales periódicas repetidas de 2.7 y 12 años (Fig. 7).

Los modelos de función de respuesta climática de los índices SOI $(33.838 \pm 0.269)$ y MEI (11.111 \pm 0.279$)$ mensuales explicaron menos la variabilidad de las cronologías de crecimiento que la de precipitación, temperatura del aire, TSM y vientos regionales.

Los índices SOI y MEI que monitorean el ENSO correlacionan inversamente con el crecimiento (Fig. 8). Ello es lógico por cuanto la fase cálida y seca del fenómeno ENSO en la región (El Niño) y la fase fría y lluviosa 


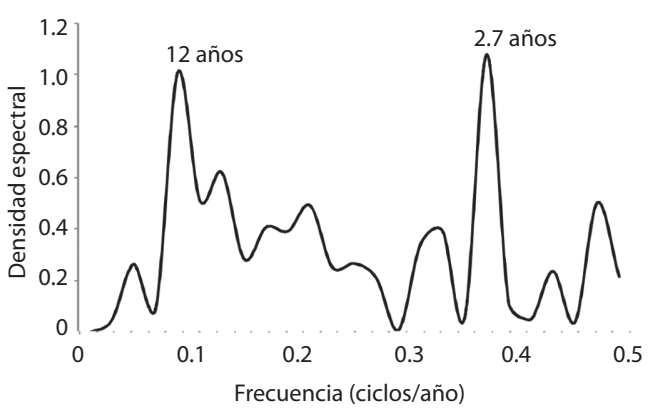

Fig. 7. Periodograma de la cronología residual de Capparis odoratissima: densidad espectral versus frecuencia.

Fig. 7. Periodogram of the residual chronology of Capparis odoratissima: spectral density versus frequency.

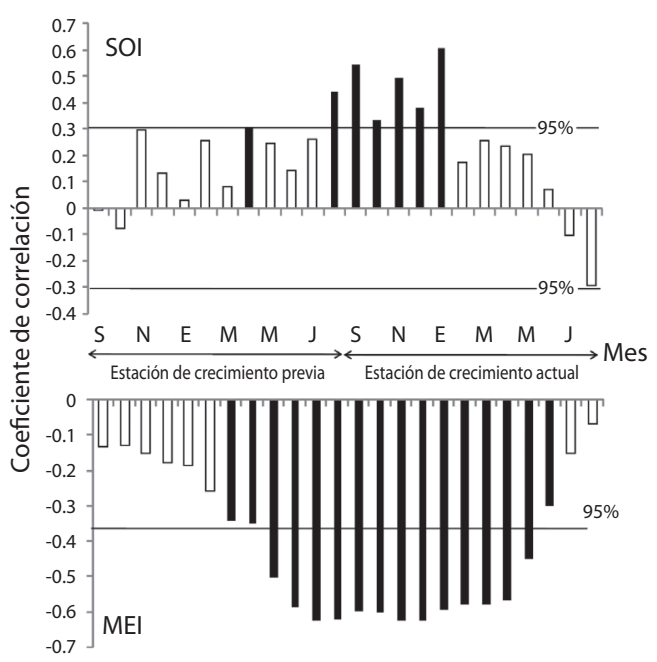

Fig. 8. Coeficientes de correlación entre la cronología residual de Capparis odoratissima con el índice SOI (izquierda) y el índice MEI (derecha). Las barras negras indican significancia para $\mathrm{p}<0.05$.

Fig. 8. Coefficients of correlation between the residual chronology of Capparis odoratissima and the Southern Oscillation Index (SOI index; left) and Multivariate ENSO Index (MEI index; right). The black bars mean significant for $\mathrm{p}<0.05$.

(La Niña) corresponden a signos contrarios en ambos índices. La fase La Niña corresponde a valores positivos del índice SOI y negativos del índice MEI, que se manifiestan en la zona por eventos de precipitación más intensos, acompañados de disminución de las temperaturas del aire y superficial del mar. Lo opuesto sucede durante la fase El Niño.

\section{DISCUSIÓN}

Las secciones deben pulirse cuidadosamente para que los anillos puedan observarse. Aunque $C$. odoratissima no es una especie decidua y probablemente continúe fotosintetizando durante la sequía, dicha reducción de la fotosíntesis conlleva tasas de crecimiento significativamente bajas que generan la marcación del anillo anualmente. Worbes (1999) reportó situaciones similares en árboles de un bosque deciduo estacional de Venezuela.

La correspondencia exacta entre los anillos predatados y el análisis de $\mathrm{C}^{14}$ es una evidencia muy convincente de la existencia de anillos anuales en la especie. Pero esta no es la única evidencia de que los anillos de crecimiento de esta especie son anuales, la datación cruzada (Crossdating en inglés) o cofechado entre los diferentes árboles es otro indicio muy fuerte de que existe crecimiento rítmico anual en todos los árboles (Eckstein et al. 1981, Schweingruber 1988, Worbes 1995). Más aun, Borchert (1999) considera que la sola correlación significativa, como la aquí encontrada (ver correlaciónes entre el ancho de los anillos y los predictores climáticos regionales arriba), entre las variables climáticas anuales con los anillos es ya una evidencia de su anualidad por cuanto esto difícilmente sucedería por azar.

La existencia de anillos de crecimiento anuales en regiones tropicales áridas o estacionalmente áridas sugiere una fase de latencia o, al menos, de baja actividad cambial inducida por la estacionalidad en la disponibilidad de agua (Mariaux 1969, 1970, Détienne \& Mariaux 1976, Borchert 1994a, Borchert 1998, Lisi et al. 2008), caracterizada por una temporada húmeda seguida por otra seca con menos de $60 \mathrm{~mm}$ mensuales de precipitación (Worbes 1995). Tales condiciones se presentan en la zona de estudio por cuanto, en promedio, entre diciembre y agosto la precipitación pluvial se mantiene por debajo de $60 \mathrm{~mm}$ mensuales 
(Fig. 1). Así, los árboles entran en estado de cuasi latencia cambial marcándose el final del anillo de crecimiento. Luego, entre septiembre y noviembre, en promedio, la humedad del suelo aportada por las lluvias desencadena una intensa actividad cambial responsable, en gran medida, del cambio del anillo. Entre más prolongada e intensa sea la temporada de lluvias durante un año en particular, más ancho será el anillo. Estos resultados concuerdan con muchos otros llevados a cabo en el Neotrópico (por ejemplo: Worbes 1989, Devall et al. 1995, Worbes 1999, Enquist \& Leffler 2001, Fichtler et al. 2003, Brienen \& Zuidema 2005, Rodríguez et al. 2005, López et al. 2005, 2006, Lisi et al. 2008). Aunque el ritmo anual de las lluvias en La Guajira es bimodal (Fig. 1), con un pico relativo de solo unos $40 \mathrm{~mm}$ durante el primer semestre en el mes de abril y otro pico absoluto de aproximadamente $120 \mathrm{~mm}$ en el mes de octubre, parece que los árboles no registran el primer pico de precipitación quizá porque éstas son tan bajas que no alcanzan a romper la latencia del cámbium o el aumento de su actividad. El efecto desecante y el aumento de la evapotranspiración que producen los vientos secos del Este de la corriente superficial de San Andrés (su nombre proviene de la isla de San Andrés en el Caribe colombiano, Poveda et al. (2006)) que soplan a más de 7m/s (Fig. 1) en el mes de abril también pueden ser responsables de la aparente inactividad del cámbium durante este máximo pluvial relativo. El hecho es que se forma un solo anillo cada año.

$C$. odoratissima presentó una fuerte correlación entre la cronología residual y las variables climáticas mensuales, como se observa en el coeficiente de determinación calculado mediante regresión múltiple sobre componentes principales de las variables climáticas mensuales y el índice de crecimiento. Ello sugiere que sus anillos son sensibles al clima. La correlación entre el ancho de los anillos y las precipitaciones mensuales fue positiva $(\mathrm{r}=0.77)$ y parece reaccionar a pequeños aumentos de precipitación. Ello se pone de presente en la correlación positiva y significativa entre la serie de los anillos y la precipitación durante el mes de agosto de la estación de crecimiento previa cuando se anticipa la temporada lluviosa, presentándose ligeras cantidades de lluvias (Fig. 5). Lo anterior sugiere relaciones fenológicas (Worbes 1989, 1999, Devall et al. 1995, Enquist \& Leffler 2001, Fichtler et al. 2003, Brienen \& Zuidema 2005, Rodríguez et al. 2005, López et al. 2005, 2006, Lisi et al. 2008). Las especies siempre verdes, como $C$. odoratissima, mantienen su follaje y transpiran durante la época de sequía gracias a su sistema radical profundo que, en ocasiones, puede alcanzar el agua de los mantos freáticos. Por el contrario, las especies deciduas tienen un sistema radical más somero (Sobrado \& Cuenca 1979, Sobrado 1986, Borchert 1994b). La presencia de anillos de crecimiento en $C$. odoratissima parece una manifestación de las limitaciones del crecimiento durante la estación seca pues, de alcanzar el agua subsuperficial, esta sólo mantiene vivas las estructuras foliares y las raíces finas.

La correlación entre la serie de crecimiento y las temperaturas medias mensuales del aire fue inferior a otras variables climáticas $(r=-$ $0.56)$ y negativas y, en muchos casos, significativas, principalmente al inicio y durante la estación lluviosa (SON, Fig. 5). Las correlaciones negativas podrían explicarse por el aumento exponencial de la transpiración causada por el aumento de la temperatura. Ello implica pérdidas altas de carbón asimilable y, por ende, de energía (Fitter \& Hay 2001). Tendencias similares encontraron Clark et al. (2003) al correlacionar la temperatura mínima diaria promedia anual con el incremento diamétrico anual de los árboles del dosel durante 16 años en Costa Rica. Llama la atención que una especie nativa de La Guajira y por tanto supuestamente adaptada a las altas temperaturas allí prevalecientes durante todo el año, presente correlaciones negativas entre el crecimiento y las temperaturas del aire (Fig. 5) durante todos los meses tanto de la estación de crecimiento corriente como la del año anterior. Ello podría significar que esta especie es muy vulnerable a los eventuales aumentos de temperatura previstos por el IPCC (2007) para estas regiones áridas. En resumen, lo anterior corrobora que el 
factor que controla el crecimiento es el balance hídrico (Borchert 1991, Lisi et al. 2008, Fig. 5). Ello sugiere que las altas temperaturas del aire actúan aumentando la evapotranspiración y reduciendo la disponibilidad de agua en el suelo y mantos freáticos. La TSM y los vientos juegan un papel preponderante en el clima de la península de La Guajira así como las oscilaciones de la Zona de Convergencia Intertropical (ZCIT; Bernal et al. 2006). En La Guajira tienen particular importancia los vientos Alisios del noreste y el Chorro Superficial de San Andrés cuya dirección predominante es hacia el Este, entre las latitudes $13^{\circ}-15^{\circ} \mathrm{N}$, responsable del clima seco y de que no exista una estación lluviosa marcada durante MAM, como sugeriría el paso de la ZCIT en esta temporada (Barriga et al. 1985, Poveda 1998, 2004, Poveda et al. 2006). Precisamente, durante la época más húmeda del año la TSM es alta y los vientos Alisios asociados con el Chorro de San Andrés alcanzan las menores velocidades del año (Fig. 5). Por el contrario, la serie de crecimiento se comporta de manera opuesta a la TSM en relación con el viento desde julio hasta noviembre (Fig. 5). Esto indicaría que a mayores TSM y menores velocidades del viento durante esta temporada, mayores serán las tasas de crecimiento asociadas con las altas precipitaciones.

Se afirma que el cambio climático global incrementará la variabilidad climática en las regiones áridas del planeta (IPCC 2007). Por otra parte, El IDEAM (2001) prevé que en La Guajira se acentuará la aridez como resultado del cambio climático. Los modelos de simulación también predicen para estas regiones aumentos de la temperatura media anual del orden de $4-5^{\circ} \mathrm{C}$ para finales del siglo XXI (Whitford 2002), simultáneamente con disminución de la humedad del suelo (Williams \& Balling 1996). Bajo el escenario A2B del IPCC las simulaciones más recientes predicen que para finales del siglo XXI la temperatura promedia de La Guajira aumentaría en $2.5^{\circ} \mathrm{C}$ y la precipitación se reduciría en más del $50 \%$ (IDEAM 2008). Desafortunadamente estos modelos de simulación arrojan resultados muy pobres en muchas regiones tropicales quizá debido a la escasa información disponible como lo han demostrado para la temperatura superficial de la tierra Christy et al. (2007), y para las precipitaciones Allan \& Soden (2008). La función de transferencia reconstruyó la precipitación total, la precipitación de la estación lluviosa (SON) y la TSM durante el período de cobertura de la serie de crecimiento de $C$. odoratissima. Ninguna de las series climáticas reconstruidas presentó indicios que indiquen tendencia significativa respecto al aumento o descenso de alguna de estas variables durante el período de estudio (Cuadro 2, Fig. 6). Ello coincide con los modelos de Bernal et al. (2006) para La Guajira, quienes encontraron evidencias de calentamiento a largo plazo en la temperatura superficial del mar en el suroeste del Caribe colombiano, más no en el noreste. Los autores atribuyen tal comportamiento a un proceso de largo plazo originado en la esquina suroeste del Caribe, asociado con la piscina cálida de Indonesia y con el giro ciclónico de Panamá-Colombia.

El ENSO es el principal mecanismo de la variabilidad climática anual en el trópico suramericano (Poveda \& Mesa 1997). ENSO presenta dos fases: El Niño y La Niña. Durante la fase El Niño se presentan anomalías positivas en la TSM, negativas en la precipitación y positivas en la temperatura del aire en el norte del Brasil, norte de la Amazonia, la Puna de Bolivia y Perú, Colombia y la costa Pacífica de Centro América. Por el contrario, el sur de Brasil y el norte del Perú exhiben condiciones pluviales anómalas durante la fase El Niño (Poveda \& Mesa 1997). Durante la fase La Niña se presentan anomalías positivas en la precipitación y negativas en la temperatura en Centro América, el Caribe y el norte de Sur América (Rogers 1988, Kiladis \& Diaz 1989). Por esta razón afirmamos que en $\mathrm{La}$ Guajira El Niño es cálido y seco y La Niña fresca y lluviosa.

Diversas cronologías de anillos de crecimiento en el trópico americano han detectado el ENSO; algunas explícitamente mencionan la fase El Niño (Stahle et al. 1998, Trouet et 
al. 2001, Fichtler et al. 2004, Schöngart et al. 2004, 2005, 2006, López et al. 2006). Hasta ahora ninguna de las investigaciones examinadas por nosotros había detectado en sus cronologías o había mencionado explícitamente la fase La Niña, tal como sucede en C. odoratissima y en Parkinsonia praecox de la misma región (Ramírez 2007).

Los índices SOI y MEI del ENSO correlacionaron significativamente con la serie de crecimiento de los anillos de $C$. odoratissima (r entre -0.33 y 0.58 para MEI y SOI, respectivamente). Las respuestas mensuales siempre positivas respecto al índice SOI y siempre negativas respecto al MEI aportan evidencia del efecto de La Niña, más no de El Niño, y son consistentes con la forma en que se calculan estos índices y ponen de presente la asociación entre el crecimiento con la fase $\mathrm{La}$ Niña. Nosotros encontramos muy lógico este resultado por cuanto en La Guajira los efectos contrastantes de las fases del ENSO alcanzan gran magnitud (IDEAM 2008); durante El Niño la temperatura aumenta más de medio grado Celsius y se reducen las lluvias en más del $40 \%$ de los promedios históricos. Durante La Niña las precipitaciones aumentan en más del $40 \%$ de los promedios históricos. Por tanto, el déficit hídrico se reduce en la región durante La Niña estimulando el crecimiento. Durante El Niño, se encuentra una acentuada aridez en los árboles con bajísima actividad fisiológica, por lo que no se detecta su efecto en la ya probablemente casi nula actividad cambial. Una situación similar se ha reportado en el desierto peruano cerca de Piura con precipitaciones anuales promedias menores de $50 \mathrm{~mm}$. Allí los anillos de Prosopis spp. y Bursera graveolens registran la fase El Niño que en esta región es lluviosa y fresca y, por tanto, equivalente a $\mathrm{La}$ Niña en La Guajira, más no detectan La Niña, más cálida y más seca en el desierto peruano (Rodríguez et al. 2005), al igual que sucede con El Niño en La Guajira.

Las correlaciones mensuales comprenden dos años calendario, iniciando en la estación previa de crecimiento (un año de rezago) y continuando durante la estación de crecimiento corriente hasta aproximadamente el mes de mayo (Fig. 8). Ello es consistente con el ciclo anual del ENSO: incremento en las anomalías de la TSM durante la primavera del hemisferio norte (estación de crecimiento previa), intensificación durante el invierno (DEF), y decaimiento hacia finales de la primavera y el verano (Poveda et al. 2006), estación de crecimiento actual. Cuando comparamos la Fig. 5 con la 8 notamos que parecen mejores predictores del crecimiento los índices globales del ENSO que las mediciones climáticas locales. Creemos que ello se debe a la asociación de un conjunto de variables meteorológicas que varían en el tiempo y en el espacio en un sólo pero significativo índice (Stenseth et al. 2003, Hallett et al. 2004).

La TSM experimenta anomalías positivas débiles durante o después de la fase cálida de El Niño en el Atlántico del trópico americano y el Caribe, especialmente durante MAM (Nobre \& Shukla 1996, Enfield \& Mayer 1997). Es decir, SOI y MEI presentan rezago de seis meses con respecto al Pacífico (Poveda \& Mesa 1997). Tal situación concuerda con las correlaciones negativas significativas mostradas en la Fig. 6 para la temperatura del aire y TSM durante marzo y abril de la estación de crecimiento corriente. Parece lógico suponer que al aumentar la temperatura también aumentarían las tasas de respiración y, por ende, disminuiría la tasa neta de asimilación del carbono.

Poveda (1998) menciona la intensificación de la velocidad del viento en los chorros atmosféricos superficiales colombianos durante los eventos El Niño en la temporada MAM. Cabalmente es en esta temporada cuando las correlaciones entre las series de crecimiento y la velocidad del viento son positivas estimulando el crecimiento a medida que aumenta la velocidad del viento. Ello, quizá, se debe al aumento de humedad transportada por la Corriente Superficial o Chorro del Chocó ( $\mathrm{Su}$ nombre proviene de la región del Chocó, Pacífico colombiano, la región más lluviosa de las Américas), al cual se le atribuye en gran medida la precipitación en el centro, oriente y norte de Colombia (Poveda et al. 2006) y 
de que el segundo semestre de estas regiones tenga un pico más alto de precipitación que el primer semestre, tal como sucede en La Guajira (Fig. 1).

ENSO es una oscilación irregular con intervalo de recurrencia entre dos y 10 años y una media de cuatro años (Trenberth 1991, 1997). El análisis espectral detectó señales periódicas en las cronologías de $C$. odoratissima con periodicidades de 2.7 y 12 años. Se considera (Poveda 2004) que las periodicidades entre 2.6 y 3.0 años son oscilaciones cuasi-bienales del ENSO. Dichos ciclos son similares a los encontrados por Cole et al. (1993) y Urban et al. (2000) en análisis espectrales de $\delta^{18} \mathrm{O}$ contenidos en anillos de arrecifes de coral y registros del SOI en la región de El Niño. En anillos de crecimiento en el trópico también se han detectado ciclos similares en la Amazonia (Schöngart et al. 2004) y en Piura y Casma, Perú (López et al. 2005, 2006). Éstos máximos parecen entonces causados por la variabilidad en la precipitación (Fichtler et al. 2004).

Urban et al. (2000) encontraron periodicidades entre 10 y 15 años asociadas con eventos ENSO, coherentes con la señal de 12 años encontrada en la serie de crecimiento de $C$. odoratissima. No obstante, no podría descartarse que corresponda con un segundo armónico de la señal de seis años encontrada por Ramírez (2007) en Parkinsonia praecox en la misma región, así como a señales de ciclo muy próximo encontradas por Schöngart et al. (2004) en la Amazonia, por López et al. (2006) en Casma, Perú, por Fichtler et al. (2004) en Namibia y por Stahle et al. (1998) en Indonesia. Pero también podría deberse al ciclo corto de 11 años de las manchas solares, tal como han sido encontradas en los anillos de Fitzroya cupressoides en Chile (Rigozo et al. 2005).

En conclusión, la naturaleza anual de los anillos de crecimiento de $C$. odoratissima en La Guajira y la posibilidad de realizar datación cruzada entre muestras de la misma especie implica un alto potencial para realizar estudios dendrocronológicos en esta parte del continente americano. Las altas correlaciones entre las series de crecimiento de dicha especie con las variables climáticas locales y globales presentadas en este trabajo, abren la posibilidad para futuras investigaciones ecológicas, climáticas, oceanográficas y sobre los efectos del cambio climático en los trópicos. Adicionalmente, permiten utilizar nuevos proxies en estas zonas para reconstruir la variabilidad de ENSO más allá de los períodos instrumentales. Los análisis de correlación sugieren que el crecimiento de la especie en La Guajira estaría promovido por pulsos en la precipitación durante eventos La Niña y, por el contrario, la fase El Niño, no la detectan tan claramente los árboles. Durante el período analizado, no se evidencia ninguna tendencia dirigida hacia el desarrollo de procesos de desertificación, representados en la disminución de la precipitación o aumento de la temperatura.

\section{AGRADECIMIENTOS}

Los autores expresan su agradecimiento a las siguientes entidades: Dirección de Investigaciones de la Universidad Nacional de Colombia sede Medellín (DIME) por la financiación parcial de la investigación, al Laboratorio de Bosques y Cambio Climático de la misma Universidad por la utilización de sus instalaciones y equipos. A la Corporación Autónoma Regional de La Guajira (CORPOGUAJIRA) por el apoyo logístico durante el trabajo de campo y al Instituto de Hidrología Meteorología y Estudios Ambientales (IDEAM) de Colombia por el suministro de la información climática.

\section{RESUMEN}

Existe gran preocupación por el efecto del cambio climático en las regiones áridas y semi-áridas tropicales. Los modelos climáticos tanto locales como globales predicen que la precipitación pluvial de la árida Península de La Guajira en el Caribe colombiano se reduciría, y las temperaturas tanto del aire como superficial del mar aumentarían como consecuencia del cambio climático. Para tratar de verificar estas hipótesis construimos una cronología del ancho de los anillos de crecimiento de Capparis odoratissima en la Media Península de La Guajira. La cronología mostró relaciones lineales estadísticamente significativas con las variables climáticas locales: temperatura del aire, temperatura superficial del mar (TSM), precipitación 
anual, velocidad del viento y variables climáticas del fenómeno ENSO (índices SOI y MEI). Las series de tiempo que calculamos mediante la función de transferencia entre 1955 y 2003, no mostraron tendencias estadísticamente significativas que demostraran que en ese período de tiempo la precipitación anual o las temperaturas han cambiado. La naturaleza anual de los anillos de crecimiento de $C$. odoratissima, la posibilidad de realizar datación cruzada entre muestras de esta especie y la alta correlación con las variables climáticas locales y globales, señalan un alto potencial de esta especie para realizar estudios dendrocronológicos en esta parte del continente americano.

Palabras clave: dendroclimatología, anillos anuales en árboles tropicales, bosques semiáridos tropicales, datación por radio-carbono, reconstrucción climática.

\section{REFERENCIAS}

Allan, R.A. \& B.J. Soden. 2008. Atmospheric warming and the amplification of precipitation extremes. Science 321: 1481-1484.

Baker, P.J. 2003. Tree age estimation for the tropics: A test from the Southern Appalachians. Ecol. Appl. 13: 1718-1732.

Barriga, E., C. Hernández, I. Jaramillo, L.E. Mora, P. Pinto \& P.M. Ruiz. 1985. La Isla de San Andrés. Contribuciones al conocimiento de su ecología, flora, fauna y pesca. Universidad Nacional de Colombia, Bogotá, Colombia.

Blasing, T.J., A.M. Solomon \& D.N. Duvick. 1984. Response functions revisited. Tree-Ring Bull. 44: 1-15.

Bernal, G., G. Poveda, P. Roldán \& C. Andrade. 2006. Patrones de variabilidad de las temperaturas superficiales del mar en la Costa Caribe Colombiana. Rev. Acad. Colomb. Cienc. 30: 195-208.

Christy, J.R., W.B. Norris, R.W. Spencer \& J.J. Hnilo. 2007. Tropospheric temperature change since 1979 from tropical radiosonde and satellite measurements. J. Geophys. Res. 112: D06102.

Borchert, R. 1991. Growth periodicity and dormancy, p. 221-245. In A.S. Raghvendra (ed.). Physiology of Trees. Wiley \& Sons, Nueva York, EEUU.

Borchert, R. 1994a. Water status and development of tropical trees during seasonal drough. Trees-Struct. Funct. 8: $115-125$

Borchert, R. 1994b. Water storage in soil or tree stems determines phenology and distribution of tropical dry forest trees. Ecology 75: 1437-1449.
Borchert, R. 1998. Responses of tropical trees to rainfall seasonality and its long-term changes. Climatic Change 39: 381-393.

Borchert, R. 1999. Climatic periodicity, phenology, and cambium activity in tropical dryforest trees. IAWA J. 20: 239-247.

Brauch, H.G. \& U.O. Spring. 2009. Securitizing the ground: grounding security, desertification, land degradation and drought. UNCCD issue paper No 2, Nueva York, EEUU. (también disponible en línea: www.unced.int/knowledge/docs/dldd_eng.pdf).

Brienen, R. 2005.Tree rings in the tropics: A study on growth and ages of Bolivian rain forest trees. PROMAB Scientific Series 10. Enchede, Utrecht, Holanda.

Brienen, R. \& P.A. Zuidema. 2005. Relating tree growth to rainfall in Bolivian rain forests: a test for six species using tree ring analysis. Oecologia 146: 1-12.

Clark, D.A., S.C. Piper, C.D. Keeling \& D.B. Clark. 2003. Tropical rain forest tree growth and atmospheric carbon dynamics linked to interannual temperature variation during 1984-2000. P. Natl. Acad. Sci. USA.100: 5852-5857.

Cole, J.E., R.G. Fairbanks \& G.T. Shen. 1993. Recent variability in the Southern Oscillation. Isotopic results from a Tarama Atoll coral. Science 260: 1790-1793.

Cook, E.R. \& L.A. Kairiukstis. 1992. Methods of dendrochronology: applications in the environmental sciences. Kluwer Academic Publishers, Dordrecht, Boston, Londres.

Cook, E.R. \& R.L. Holmes. 1996. User's manual for program ARSTAN. Laboratory of Tree-Ring Research. University of Arizona, Tucson, EEUU.

Détienne, P. \& A. Mariaux. 1976. Nature et périodicité des cernes dans les bois de Samba. Bois For. Trop. 169: 29-35.

Devall, M.S., B.R. Parresoll \& S.J Wright. 1995. Dendroecological analysis of Cordia alliodora, Pseudobombax septenatum and Annona spraguel in central Panama. IAWA J. 16: 411-424.

Eckstein, D., J. Ogden, G. Jacoby \& J. Ash. 1981. Age and growth rate determination in tropical trees: the application of dendrochronological methods, p. 83-106. In F. Bormann \& G. Berlyn (eds.). Age and growth rate of tropical trees: New directions for research. Yale University, New Haven, EEUU.

Enfield, D.B. \& D.A. Mayer. 1997. Tropical Atlantic sea surface temperature variability and its relation to $\mathrm{El}$ 
Niño-Southern Oscillation. J. Geophys. Res. 102: 929-946.

Enquist, D.B. \& J. Leffler. 2001. Long-term tree ring chronologies from sympatric tropical dry-forest trees: individualistic responses to climatic variation. J. Trop. Ecol. 17: 41-60.

Fichtler, E., D.A. Clark \& M. Worbes. 2003. Age and long-term growth of trees in an old-growth tropical rain forest, based on analyses of tree rings and ${ }^{14} \mathrm{C}$. Biotropica 35: 306-317.

Fichtler, E., V. Trouet, H. Beeckman, P. Coppin \& M. Worbes. 2004. Climatic signals in tree rings of Burkea Africana and Pterocarpus angolensis from semiarid forests in Namibia. Trees-Struct. Funct. 18: 442-451.

Fitter, A.H. \& R.K.M. Hay. 2002 . Environmental physiology of plants. Academic, Londres, Reino Unido.

Fritts, H.C. 1976. Tree rings and climate. Academic, Londres, Reino Unido.

Grainger, A., M. Stafford-Smith, V. Squires \& E. Glenn. 2000. Desertification and climate change: the case for greater convergence. Mitig. Adapt. Strateg. Glob. Change 5: 361-377.

Grissino-Mayer, H.D. 2001. Evaluating crossdating accuracy: a manual and tutorial for thecomputer program COFECHA. Tree-Ring Res. 57: 205-221.

Hallett, T.B., T. Coulson, J.G. Pilkington, T.H. CluttonBrock, J.M. Pemberton \& B.T. Grenfell. 2004. Why large-scale climate indices seem to predict ecological processes better than local weather. Nature 430: 71-75.

Holmes, R.L. 1983. Computer-assisted quality control in tree-ring dating and measurement. Tree-Ring Bull. 43: 69-78.

Hua, Q. \& M. Barbetti. 2004. Review of tropospheric bomb $14 \mathrm{C}$ data for carbon cycle modeling and age calibration purposes. Radiocarbon 46: 1273-1298.

IDEAM, MAVDT \& PNUD. 2001. Colombia, Primera comunicación nacional ante la convención marco de las Naciones Unidas sobre el Cambio Climático. IDEAM, Bogotá, Colombia.

IDEAM, MAVDT \& PNUD. 2008. Reflexiones sobre el clima futuro y sus implicaciones en el desarrollo humano en Colombia. PNUD, Bogotá, Colombia (también disponible en línea: http://www.pnud.org. co/sitio.shtml?apc=a-c020011--\&x=18641).
Kalnay, E., M. Kanamitsu, R. Kistler, W. Collins, D. Deaven, L. Gandin, M. Iredell, S. Saha, G. White, J. Woollen,Y. Zhu, M. Chelliah, W. Ebsuzaki, W. Higgins, J. Janowia, K.C. Mo, C. Ropelewski, J. Wang, A. Leetma, R. Reynolds, R. Jenne \& D. Joseph. 1996. The NCEP/NCAR 40-year reanalysis project. B. Am. Meteorol. Soc.77: 437-470.

Kiladis, G.N. \& H.F. Diaz. 1989. Global climatic anomalies associated with extremes in the Southern Oscillation. J. Climate 2: 1069-1090.

Lamprecht, H. 1990. Silvicultura en los trópicos. GTZ, Eschborn, Alemania.

Le Houérou, H.N. 1996. Climate change, drought and desertification. J. Arid Environ. 34: 133-185.

Lieberman, D., M. Lieberman, G. Hartshorn \& R. Peralta. 1985. Growth rates and age- size relationships of tropical wet forests trees in Costa Rica. J. Trop. Ecol. 1: 97-109.

Lisi, C.S., M. Tomazello, S.A. Botosso, F.A. Roig, V.R.B. María, L. Ferreira-Feselle \& A.R.A Voigh. 2008. Tree-ring formation, radial increment periodicity, and phenology of tree species from a seasonal semideciduous forest in Southern Brazil. IAWA J. 29: 189-208.

López, B.C., S. Sabate, C.A. Gracia \& R. Rodríguez. 2005. Wood anatomy, description of annual rings, and responses to ENSO events of Prosopispallida H.B.K., a wide-spread woody plant of arid and semi-arid lands of Latin America. J. Arid Environ. 61: 541-554.

López, B.C., R. Rodríguez, C.A. Gracia \& S. Sabate. 2006. Climatic signals in growth and its relation to ENSO events of two Prosopis species following a latitudinal gradient in South America. Glob. Change Biol. 12: 897-906.

Lyon, B. 2004. The strength of El Niño and the spatial extent of tropical drought. Geophys. Res. Lett. 31: L21204.

Mariaux, A. 1969. La périodicité des cernes dans les bois de Limba. Bois For. Trop. 128: 39-54.

Mariaux, A. 1970. La périodicité de formation des cernes dans les bois de l'Okoumé. Bois For. Trop. 131: 37-50.

NCAR. The National Center for Atmospheric Research. Southern Oscillation Index (SOI). (Consultado: 19 octubre 2007, http://www.cgd.ucar.edu/cas/catalog/ climind/soi.html). 
New, M., M. Todd, M. Hulme \& P. Jones. 2001. Precipitation measurements and trends in the twentieth century. Int. J. Climatol. 21: 1843-1950.

NOAA. National Oceanic and Atmospheric Administration, Earth System Research Laboratory. (Consultado: 19 octubre 2007, http://www.esrl.noaa.gov/psd/ people/klaus.wolter/MEI/).

Nobre, P. \& J. Shukla. 1996. Variations of sea surface temperature, wind stress and rainfall over the Tropical Atlantic and South America. J. Climate 9: 2464-2479.

Poveda, G. 1998. Retroalimentación dinámica entre el fenómeno El Niño-Oscilación del Sur y la hidrología de Colombia. Tesis de Doctorado, Universidad Nacional de Colombia Sede Medellín, Colombia.

Poveda, G. 2004. La hidroclimatología de Colombia: una síntesis desde la escala inter-decadal hasta la escala diurna. Rev. Acad. Colomb. Cienc. Exactas, Fis. Nat. 28: 201-222.

Poveda, G., P.R. Waylen \& R.S. Pulwarty. 2006. Annual and inter-annual variability of the present climate in northern South America and southern Mesoamerica. Palaeogeogr. Palaeocl. 234: 3-27.

Poveda, G. \& O.J. Mesa. 1997. Feedbacks between hydrological processes in tropical South America and largescale ocean-atmospheric phenomena. J. Climate 10: 2690-2702.

Ramírez, J.A. 2007. Relación entre la señal climática de cronologías de Capparis odoratissima y Cercidium praecox con la variabilidad climática local y global de La Guajira, Colombia. Tesis Maestría, Universidad Nacional de Colombia Sede Medellín, Colombia.

Reimer, P.J., T.A. Brown \& R.W. Reimer. 2004. Discussion: Reporting and calibration of post-bomb ${ }^{14} \mathrm{C}$ data. Radiocarbon 46: 1299-1304.

Reynolds, R.W. \& T.M. Smith. 1994. Improved global sea surface temperature analyses using optimum interpolation. J. Climate 7: 929-948.

Rigozo, N.R., D.F.R. Nordeman, E. Echer, L.E.A. Vieire, M.P.S. Echer \& A. Prestes. 2005. Tree-ring width wavelet and spectral analysis of solar variability and climatic effects on a Chilean cypress during the last two and a half millennia. Clim. Past 1: 121-135.

Rodríguez, R., A. Mabresa, B. Luckman, M. Masiokas \& T.M. Ektvedt. 2005. "El Niño" events recorded in dry-forest species of the lowlands of northwest Peru. Dendrochronologia 22: 181-186.
Rogers, J.C. 1988. Precipitation variability over the Caribbean and tropical Americas associated with the Southern Oscillation. J. Climate 1: 172-182.

Roig, F. 2000. Dendrocronología en América Latina. EDIUNC, Mendoza, Argentina.

Rozendaal, D.M.A. 2010. Looking backwards: using tree rings to evaluate long-term growth patterns. Ph.D. Thesis, Utrecht University, Enschede, Utrecht, Holanda.

Schöngart, J., B. Orthmann, K.J. Hennenberg, S. Porembski \& M. Worbes. 2006. Climate-growth relationships of tropical tree species in West Africa and their potential for climate reconstruction. Glob. Change Biol. 12: 1139-1150.

Schöngart, J., M.T.F. Piedade, F. Wittmann, W.J. Junk \& M. Worbes. 2005. Wood growth patterns of Macrolobiumacaciifolium (Benth.) Benth. (Fabaceae) in Amazonian black-water and white-water floodplain forests. Oecologia 145: 454-461.

Schöngart, J., W.J. Junk, M.T.F. Piedade, J.M. Ayres, A. Hüttermann \& M. Worbes. 2004. Teleconnection between tree growth in the Amazonian floodplains and the El Niño-Southern Oscillation effect. Glob. Change Biol. 10: 683-692.

Schweingruber, F. 1988. Tree rings: basis and applications of dendrochronology. Reidel, Dordrecht, Holanda.

Sobrado, M.A. 1986. Aspects of tissue water relations and seasonal changes of leaf waterpotential components of evergreen and deciduous species coexisting in tropical dry forests. Oecologia 68: 413-416.

Sobrado, M.A. \& G. Cuenca. 1979. Aspectos del uso de agua de especies deciduas y siempreverdes en un bosque seco tropical de Venezuela. Acta Cient. Venez. 30: 302-308.

Solomon, S., D. Qin, M. Manning, Z. Chen, M. Marquis, K.B. Averyt, M. Tignor \& H.L. Miller. Climate change 2007: the physical science basis: contribution of working group I to the fourth assessment report of the intergovernmental panel on climate change. Cambridge, Nueva York, EEUU.

Stahle, D.W., R.D. D'Arrigo, P.J. Krusic, M.K. Cleaveland, E.R. Cook, R.J. Allan, J.E. Cole, R.B. Dunbar, M.D. Therrell, D.A. Gay, M.D. Moore, M.A. Stokes, B.T. Burns, J. Villanueva-Diaz \& L.G. Thompson. 1998. Experimental dendroclimatic reconstruction of the Southern Oscillation. B. Am. Meteorol. Soc. 79: 2137-2152. 
Stenseth, N.C., G. Ottersen, J.W. Hurrell, A. Mysterud, M. Lima, K. Chan, N.G. Yoccoz \& B.A. Ådlandsvik. 2003. Studying climate effects on ecology through the use of climate indices: the North Atlantic Oscillation, El Niño Southern Oscillation and beyond. P. Roy. Soc. Lond. B. Bio. 270: 2087-2096.

Trenberth, K.E. 1984. Signal versus noise in the Southern Oscillation. Mon. Weather Rev. 112: 326-332.

Trenberth, K.E. 1991. General characteristics of El NiñoSouthern Oscillation, p. 13- 42. In Teleconnections linking worldwide climate anomalies. R.M. Glantz, R. Katz, R. Nicholls (eds.). Cambridge University, Londres, Inglaterra.

Trenberth, K.E. 1997. The definition of El Niño. B. Am. Meteorol. Soc. 78: 2771-2777.

Trouet, V., K. Haneca, P. Coppin \& H. Beeckman. 2001. Tree ring analysis of Brachystegiaspiciformis and Isoberliniatomentosa: evaluation of the ENSO-signal in the Miombo Woodland of Eastern Africa. IAWA J. 22: 385-399.

Urban, F.E., J.E. Cole \& J.T. Overpeck. 2000. Influence of mean climate change on climate variability from a 155 year tropical Pacific coral record. Nature 407: 989-993.

Whitford. W. 2002. Ecology of deserts systems. Academic, San Diego, EEUU.
Whitmore, T.C. 1975. The rain forest of the Far East. Clarendon, Oxford, Reino Unido.

Williams, M.A.J. \& R.C. Balling. 1996. Interactions of desertification and climate. Edward Arnold, Londres, Reino Unido.

Wolter, K. \& M.S. Timlin. 1998. Measuring the strength of ENSO events: how does 1997/98 rank? Weather 53: 315-324.

Worbes, M. 1989. Growth rings, increment and age of trees in inundation forests, savannas and a mountain forest in the Neotropics. IAWA Bull. 10: 109-122.

Worbes, M. 1995. How to measure growth dynamics in tropical trees, a review. IAWA J. 16: 337-351.

Worbes, M. 1999. Annual growth rings, rainfall-dependent growth and long-term growth patterns of tropical trees from the Caparo Forest Reserve in Venezuela. J. Ecol. 87: 391-403.

Worbes, M. 2002. One hundred years of tree-ring research in the tropics-a brief history and anoutlook to future challenges. Dendrochronologia 20: 217-231.

Worbes, M. \& W.J. Junk. 1999. How old are tropical trees? The persistence of a myth. IAWA J. 20: 255-260.

Worbes, M., R. Staschel, A. Roloff \& W.J. Junk. 2003. Tree ring analysis reveals age structure, dynamics and wood production of a natural forest stand in Cameroon. Forest Ecol. Manag. 173: 105-123. 